\title{
Western Balkans' Accession to the European Union, Intertwined Interests
}

\section{Edlira Titini}

\author{
Doi:10.5901/ajis.2014.v3n3p406
}

\author{
University "Aleksandër Xhuvani", Elbasan, Albania
}

\begin{abstract}
The accession of the Western Balkans to the European Union will be the completion of a process initiated in November 2000 at the Zagreb Summit, which is proceeding at a slow pace and in different ways through individual negotiations with each of the countries of this region. In the first part of this study, there will be highlighted both the reasons why the EU member states are in favor of accession of the Western Balkans to the European Union and the rumors to the contrary within the EU member countries. In the second part, there will be continued with detecting the reasons why the governments of the countries of the Western Balkans region aspire the integration in the European Union without forgetting the opposition to the EU in certain countries of the Western Balkans. At the conclusion of this study, there will be clear that the advantages of different character drawn from this process are common interests of parties, EU and Western Balkan countries.
\end{abstract}

Keywords: Balcani occidentali, potenziali candidati, Unione Europea, integrazione regionale, allargamento dell'UE.

\section{Introduzione}

Al fine di sottolineare l'approccio regionale, nel 1996 l'Unione Europea ha fatto una distinzione tra i due termini "Europa sud-orientale" e "Balcani occidentali". II termine "Europa sud-orientale" si riferisce a tutti i paesi dal Golfo di Trieste al Mar Nero, mentre la regione dei Balcani occidentali è costituito dagli stati dell'ex Jugoslavia, eccetto la Slovenia, più l'Albania'(Vesnic-Alujevic.2012).

Negli anni Novanta, le relazioni dell'Unione Europea con i paesi dei Balcani occidentali erano incentrate sulla gestione della crisi e sulla ricostruzione, in linea con le urgenti necessità dei paesi a seguito della dissoluzione della Jugoslavia. Tuttavia, emerse presto la necessità di una strategia a più lungo termine. Nel 2000, in occasione del vertice di Zagabria, I'UE avviò ufficialmente il processo di stabilizzazione e associazione (PSA), un quadro per le relazioni tra I'UE e i paesi nella regione, nonché il patto di stabilità, una più ampia iniziativa che coinvolse tutti i principali attori internazionali. II Consiglio europeo di Santa Maria da Feira (19-20 giugno 2000), dichiarò che tutti i paesi del PSA erano potenziali candidati all'adesione all'UE, posizione ribadita al Consiglio europeo di Salonicco del 2003, dove fu addirittura delineata una specifica strategia per l'inclusione nell'Unione Europea dei paesi dei Balcani occidentali.

L'Integrazione regionale dei Balcani occidentali e l'integrazione dei Balcani occidentali in Europa rappresenta un fattore di stabilità economica e politica che è nell'interesse di tutti.

Qualche anno fa la Commissione internazionale per i Balcani Occidentali, presieduta da Giuliano Amato, aveva avanzato la proposta di ingresso dei paesi dei Balcani per la data simbolica del 2014, esattamente un secolo dopo lo scoppio della Prima guerra mondiale. Nel frattempo, Slovenia, Romania, Bulgaria e Croazia sono entrate a pieno titolo nell'Unione, ma per il resto dei Balcani i tempi sembrano molto più lunghi.

Negli ultimi anni questo progetto ha subito rallentamenti e soste che spingono tutte le leadership politiche europee a prendere di nuovo in considerazione sia la natura che l'Europa dovrebbe avere sia anche e soprattutto i confini dell'Europa. Per comprendere meglio la questione dell'integrazione dei Balcani Occidentali nell'UE dobbiamo tornare indietro di alcuni decenni alla scomparsa di Josip Broz Tito, presidente della ex Jugoslavia. Con la morte di Tito nel 1980, scomparve anche il progetto, l'identità che i slavi del sud avevano maturato ma non assorbito fino in fondo poiché subito erano riaffiorati gli "egoismi" nazionali dei vari Stati, delle varie etnie, dei vari credi religiosi, dei vari e diversi mondi culturali della Jugoslavia (Brancaccio, 2006). La tensione era precipitata sconvolgendo la Federazione Jugoslava e trascinando le popolazioni nella tragedia della guerra, del genocidio e degli estremismi nazionalistici. La Guerra fratricida ha portato a circa trecentomila morti, all'impoverimento dei paesi che facevano parte della Federazione Jugoslava ma anche e soprattutto ha trasformato in oddio profondo i rapporti assai fragili tra le diverse etnie della ormai ex Jugoslavia. In quella occasione l'Europa si è dimostrata miope per capire la gravità della situazione, egoista e incapace di intervenire. Il conflitto nella ex Jugoslavia è stato un banco di prova molto importante e difficile per L'Unione Europea ma allo stesso tempo ha rappresentato la forza motrice dell'integrazione europea. Dopo il conflitto del Kosovo, la situazione era molto delicata e continuava ad imporre all'UE e alla sua politica estera notevoli esigenze. In primo luogo diventava necessario 
utilizzare correttamente l'assistenza finanziaria e tecnica dell'Unione, nel lavoro di ricostruzione post-bellica; In secondo luogo, I'UE dovrebbe essere in grado di esercitare una politica di prevenzione dei conflitti e di gestione delle crisi per la quale non aveva ancora le risorse. Le controversie che rimanevano da risolvere erano molto complesse e multidimensionali e, quindi difficili sfide per la politica estera dell'UE, ancora in fase embrionale.

\section{L'interesse dell'UE Verso i Balcani Occidentali}

Dopo la caduta del muro di Berlino, furono gli Stati Uniti a svolgere un ruolo di "guida" nei Balcani, con i contestati accordi di Dayton, che nel novembre del 1995 misero fine ufficialmente alla guerra civile in Jugoslavia, e con il successivo intervento militare in Kosovo. Dalla fine degli anni novanta, però, agli Stati Uniti è subentrata sempre di più l'Unione Europea. Alla fine dei bombardamenti sulla ex Repubblica Federale della Jugoslavia, l'UE si rese conto che la stabilità dei Balcani era una condizione della sicurezza in Europa e ritenne necessario l'adozione del Patto di Stabilità.

Quest'ultimo mira a rafforzare la pace, la democrazia, il rispetto dei diritti umani e lo sviluppo economico per raggiungere la stabilità ed il progresso in tutta la regione.

Per prevenire ulteriori possibili conflitti e promuovere la cooperazione regionale l'UE ha offerto ai paesi dei Balcani Occidentali una chiara prospettiva europea in cambio di una serie di riforme.

L'elenco delle riforme necessarie conteneva molti elementi di quello che viene chiamato "state building", tra cui la riforma dello stato di diritto, la costruzione di istituzioni stabili e democratiche, muovendosi verso un economia di mercato. Gli strumenti dell'UE a disposizione dei paesi dei Balcani Occidentali per la realizzazione di queste riforme includeva programmi di assistenza per la riabilitazione post-conflitto e la stabilizzazione, programmi di assistenza tecnica e dei programmi volti ad armonizzare la legislazione con l'acquis communautaire dell'Unione Europea. Un altra esigenza individuata dall'Unione Europea è stato il rafforzamento delle relazioni bilateriali tra i paesi della regione Balcanica, nella convinzione che ciò aprirebbe la strada per una maggiore stabilità e sviluppo politico ed economico. Un altro problema identificato è stato il bisogno di flessibilità nell'approccio dell'UE, al fine di soddisfare le esigenze specifiche di ciascun paese.

II grande impegno dell'UE e la sua nuova politica verso i Balcani occidentali dimostra il suo interesse rispetto all'integrazione di questa regione nell'UE.

Le ragioni a favore dell'adesione dei Balcani Occidentali nell'UE, come soprammenzionato, riguardano principalmente la sicurezza, ma non soltanto. Queste ragioni sono anche di natura economica, geostrategica e di prestigio dell'UE.

I Balcani Occidentali lasciati al loro destino, senza l'incentivo deciso di una futura integrazione, sarebbero molto probabilmente guidati da regimi autocratici, una fonte non trascurabile di immigrazione clandestina verso l'UE, un canale per il narcotrafico, il trafico di esseri umani e altri trafici illeciti e una "lavanderia" per il denaro sporco. La storia dei conflitti etnici, accompagnati anche da problemi delle frontiere e da violazioni dei diritti dell'uomo, che e un déjà vu per questa regione, si ripetterebbe fino a portare ad un nuovo catastrofo con ripercussioni globali. La sicurezza dell'Europa, e il suo prestigio ne pagherebbe le conseguenze. Inoltre, non sarebbe neanche realistico pensare che l'Europa può prosperare quando tiene al suo interno una regione in queste circostanze che è allo stesso tempo un crocevia dove si incontrano tre potenti forze geopolitiche: Occidente, Islam e Russia, soprattutto alla luce degli ultimi eventi in Ucraina.

Dall'adesione degli Stati dei Balcani Occidentali guadagnerebbe anche l'economia dell'Unione. Come è ovvio, più grande è il mercato unico, maggiore è la portata economica dell'UE nel mondo. La possibile delocalizzazione delle imprese europee nei nuovi Stati membri aiuterebbe le imprese, con sede nei vecchi Stati membri, a rimanere competitive sui mercati mondiali. Questa competitività incentiverebbe e consentirebbe alle imprese di restare in Europa anziché trasferire le proprie attività in altri continenti.

Nonostante i molteplici argomenti a favore dell'adesione dei paesi dei Balcani Occidentali nell'UE, con l'inizio della crisi economica e finanziaria, a partire dal 2008, UE ha rallentato il processo di adesione e non considera più una priorità la questione dei Balcani Occidentali. I cittadini e i leader dell'UE sono diventati più cauti rispetto ad ulteriori allargamenti (Szolucha,2010). La diminuzione dell'entusiasmo generale nei confronti dell'Europa ha portato con sé dei timori più 0 meno realistici e fondati ma che comunque hanno influenza sulla realtà sociale, economica, politica e culturale come: il timore che la manodopera a basso costo tolga lavoro e diritti agli attuali disoccupati europei; il timore di un aumento di corruzione e criminalità; il timore di perdere diritti di godere di aiuti strutturali ed agricoli per l'aumento degli aventi diritto e dei richiedenti; il timore di perdere potere decisionale in Europa; timore di perdere l'identità culturale, linguistica e religiosa. Però, malgrado i timori associati al processo di adesione dei Balcani occidentali, bisogna ammettere che in un mondo globalizzato e interconnesso, gli strumenti indispensabili e vincenti sono apertura reciproca e collaborazione, non chiusura e isolamento. In più i diritti si difendono promuovendoli ed estendendoli, non dividendo chi li possiede da chi no. 


\section{L'obiettivo Europeo dei Balcani Occidentali}

Dopo i conflitti balcanici degli anni novanta l'UE ebbe l'occasione per dimostrare di essere qualcosa in più di un semplice accordo comerciale. Nei confronti dei Balcani occidentali l'Unione scelse di sviluppare un approccio guidato da una logica integrazionista centrata sulla prospettiva di integrazione tra i paesi balcanici e l'Unione Europea. Nel giugno del 1999 quest ultima lanciò il Patto di Stabilità per l'Europa sud orientale attraverso cui fu proposto un nuovo approccio alla risoluzione dei conflitti nei Balcani, con una visione di lungo periodo e di intervento simultaneo nei diversi settori della sicurezza, dell'economia e dello sviluppo democratico, nella convinzione che queste dimensioni fossero fortemente interconnesse. Subito dopo è stato lanciato il Processo di Stabilizzazione e Associazione, la cornice principale dei rapporti tra l'Unione Europea e Balcani occidentali. Sia il Patto di Stabilità sia il Processo di Stabilizzazione e Associazione contengono riferimenti alla "prospettiva europea" dei Balcani occidentali.

Nel 2000 il Consiglio di Santa Maria da Feira ha indicato esplicitamente che gli strumenti posti in essere a livello dell'UE nelle relazioni con i Balcani occidentali mirano all'obiettivo dell'integrazione di questi paesi "nel contesto politico ed economico dell'Unione". In quell'occasione il Consiglio ha conferito a tutti i paesi dei Balcani occidentali la qualità di "potenziali candidati" all'adesione all'Unione1(Rizzo, 2010).

La prospettiva europea dovrebbe costituire un incentivo straordinario per trasformare, in senso favorevole alla stabilità europea, le dinamiche istituzionali, economiche, politiche e sociali di ciascuno dei paesi dei Balcani occidentali. La prospettiva europea non era e non è soltanto il futuro che UE riserva a questi paesi, ma innanzitutto quello che i governi dei Balcani occidentali e le loro popolazioni auspicano da tempo per il loro futuro.

Nonostante i problemi politici interni, i conflitti e le crisi sociali, l'obiettivo dell'integrazione non viene messo in discussione da nessun governo della regione, almeno pubblicamente. Neanche la crisi economica e politico-istituzionale dell'UE sembra aver intaccato gli obiettivi europei dei paesi candidati, 0 aspiranti tali, all'ingresso nell'UE.

L'adesione all'UE viene considerata dai paesi dei Balcani Occidentali come il modo migliore per raggiungere la prosperità economica, fermo restando le capacità di ciascuno dei paesi di usare questa opportunità. Inoltre diventare parte dell'UE offrirà un alto livello di protezione dei diritti dell'uomo e allo stesso tempo significherà pace e stabilità in un area che in passato è stata attraversata da molte guerre sanguinose.

L'adesione all'UE renderà più facili le relazioni economiche tra vecchi e nuovi membri dell'Unione e le rafforzerà a beneficio di entrambe le parti. Entrare a far parte di un mercato unico europeo porterà con se dei benefici come la libera circolazione delle persone, delle merci, dei servizi e dei capitali, maggiore concorrenza leale, maggiore scelta per i consumatori, prezzi inferiori, maggiore sicurezza del mercato data dalla normativa comune europea, maggiori investimenti esteri, aumento dell'occupazione, della produttività e degli scambi con conseguente miglioramento delle condizioni di vita delle popolazioni ecc.

Benché sia evidente che i benefici sopra elencati sono di un importanza vitale per tutti i paesi dei Balcani occidentali, è pur vero che non sono mancati gli oppositori all'adesione all'UE, come per esempio nel caso della Croazia sono state organizzate diverse manifestazioni per avvertire che il paese potrebbe cedere la propria sovranità nazionale alla burocrazia di Bruxelles. Secondo il fronte del no, la UE non sarebbe una democrazia rappresentativa, ma una fortezza burocratica in cui il Consiglio obbediente firma tutte le leggi predisposte dalla Commissione. Costoro hanno espresso anche la preoccupazione per possibili eventi quali la riduzione della produzione agricola interna, il blocco ai croati a lavorare nell'UE e la vendita agli stranieri dell'intera costa adriatica del Paese ${ }^{2}$. Anche i Serbi hanno espresso i loro timori e la loro contrarietà all'avanzamento del Processo di stabilizzazione e associazione poiché secondo loro l'Europa condiziona questo processo con il riconoscimeto dell'indipendenza del Kosovo, cosa inaccettabile per Belgrado ${ }^{3}$.

\section{Conclusioni}

Per concludere, l'allargamento dell'Unione Europea era stato previsto e voluto sin dall'origine con l'intento di costruire un Europa forte e unita nella diversità che portasse avanti i valori democratici su cui è stata fondata.

\footnotetext{
1 vedi anche le Conclusioni della Presidenza, Consiglio Europeo di Santa Maria da Feira, 19 e 20 giugno 2000 , http://www.consilium.europa.eu/ueDocs/cms_Data/docs/pressData /it/ec/00200-r1. \% 20 ann 1. i0.pdf

${ }^{2}$ vedi anche: http://www.lastampa.it/2012/01/23/blogs/voci-globali/croazia-si-alla-ue-tra-forti-contrasti-e-assenteismo-x5yoCDfSo3mP3i 4SgJTwsN/pagina.html

${ }^{3}$ vedi anche: Dacic: riconoscimento del Kosovo è la condizione inaccettabile(05.06.2013- ore19:35)-Radio Internazionale di Serbia: http://voiceofserbia.org/it/content/dacic-riconoscimento-del-kosovo-\%C3\%A8-la-condizione-inaccettabile
} 
Se da un lato si può dire che l'adesione dei paesi dei Balcani occidentali può fornire nuove energie all'Unione, soprattutto davanti alla crisi economica in corso e prevenire la diffusione di fattori di instabilità, dall'altro occorre tenere presente che l'UE è l'unica via per i Paesi dei Balcani rimasti ancora fuori dell'Unione di avere una crescita economica più rapida e di essere un giorno all'altezza del resto di Europa. occidentali.

In poche parole i Balcani occidentali hanno tanto bisogno dell'UE quanto quest'ultima ha bisogno dei Balcani

\section{Bibliografia}

Brancaccio,G., (2006). La disgregazione jugoslava e la questione albanese negli anni novanta. L'Adriatico e l'Europa centroorientale:relazioni storico- culturali e prospettive di sviluppo": lezioni della Summer school in Adriatic Studies, Bologna: CLUEB.

Dacic: riconoscimento del Kosovo è la condizione inaccettabile. (5 giu 2013, 19:35). Radio Internazionale di Serbia.

Fuentes, J., (Nov./Dic. 2003). Los Balcanes Occidentales: ¿Sexta ampliación de la UE?.

Politica exterior,17 (96). Estudios de politica Exterior S.A.

Intra, E., Parrella, B., (23 gen. 2012). Croazia: sì alla UE, tra forti contrasti e assenteismo. La Stampa.

Johansson, E., Solé i Padró, M.,(2006)Los balcanes Occidentales: ¿Tema de oportunismo español? .

Klintis, A. (1997). The EU's foreign policy and the war in former Yugoslavia' in M. Holland (ed) The Common foreign and Security Policy: The Record and Reforms, London: Pinter.

Mammarela, G., Cacace, P., (2000). Le sfide dell'Europa, attualità e prospettive dell'integrazione. Roma-Bari: Laterza Osservatorio Balcani e Caucaso. Allargamento dell'UE ai Balcani e alla Turchia. (21 nov. 2013).

Rey, B., (2013). Note sintetiche sull'Unione Europea-2013.http://www.europarl.europa.eu/RegData/etudes/fiches_techniques/2013/ 060502/04A_F\%282013\%29060502_IT.pdf

Rizzo, A., (2010). II pluralismo nella transizione costituzionale dei Balcani: diritti e garanzie. Quaderni del Dipartimento di Scienze Giuridiche, 92, Università degli Studi di Trento,

Schenker, H., (2008). The Stabilization and Association process: An Engine of European Integration in Need of Tuning. Journal on Ethnopolitics and Minority Issues in Europe (JEMIE),7, European Centre for Minority Issues.

Sjursen, H., ( 1998). Enlargement and the common foreign and security policy: transforming the EU's external policy?. Oslo: ARENA Centre for European Studies.

Szołucha, A., (2010). The EU and Enlargement Fatigue: Why has the European Union not been able to counter enlargement fatigue? Journal of Contemporary European Research, , 6 ( 1), pp. 1.

Vachudova, M. A. (2014). EU leverage and national interests in the Balkans: The puzzles of enlargement ten years on. Journal of Common Market Studies, 52(1).

Verluise, P., (2013). Géopolitique des frontiers Européennes, Institut de Relationes Internationales et Stratégiques (IRIS).

Vesnic-Alujevic, L.,(2012). European Integration of Western Balkans: From Reconciliation to European Future. Centre for European Studies.

Wulzer, P., (14 lug. 2003). Le relazioni internazionali dell'Unione Europea: dalla Cooperazione Politica Europea alla Politica Estera e di Sicurezza Comune. Storia del Mondo.Periodico telematico di Storia e Scienze Umane, 12.

Zahariadis, Y., (2007). The effects of the Albania-EU Stabilization and Association Agreement: economic impact and social implication. London: Overseas Development Institute. 